\title{
The Expressions of P53, MDM2 in Trophoblasts of Spontaneous Abortion Mouse Model and the Relevant Researches
}

\author{
Chunlan Zhou, Qian Wang, Menghuan Zeng, Liping Cheng, Xiaoyun Xie* \\ Jiaxing University, Jiaxing City, China \\ Email: 863940465@qq.com,87354674@qq.com,"99436362@qq.com
}

Received 2013

\begin{abstract}
Objective: To explore the mRNA expression of the related genes of p53, MDM2, vascular endothelial growth factor (VEGF) and hypoxia inducible transcription factors-1 a (HIF-la) in villous samples of spontaneous abortion mouse models and normal pregnancy models, and to discuss the effect of p53, MDM2 on the growth of villous trophoblast cells. Methods: The abortion-prone CBAXDBA/2 matings were established as the model of spontaneous abortion and the non-abortion-prone CBAXBALB/c matings as the model of normal pregnancy. Applied q Real-time PCR method to detect the mRNA expression levels of p53, MDM2, VEGF and HIF-la in villous samples of spontaneous abortion mouse models and normal pregnancy models. Results: The relationship of the mRNA expression level of p53, MDM2, VEGF and HIF-la in vinous samples of spontaneous abortion mouse models: in the villous samples of spontaneous abortion mouse models, the expression of p53 was positively correlated with the expression of MDM2, HIF-la $(r=0.35$; $r=0.63)$, and the relationship was significant $(P=0.01$; $P<0.001)$; but negatively correlated to the expression of VEGF $(r=-0.30)$, and the relationship was significant $(P=0.03)$. The expression of MDM2 was positively correlated with the expression of HIF-la $(r=0.28)$, and the relationship was significant $(\mathrm{P}=0.04)$; and negatively correlated with the expression of VEGF $(\mathrm{r}=-0.08)$, but the relationship was not significant $(\mathrm{P}=0.57)$. The expression of HIF-la was negatively correlated with the expression of VEGF $(r=-0.37)$, and the relationship was significant $(P=0.007)$. The relationship of the mRNA expression level of p53, MDM2, VEGF and HIF-1 a in vinous samples of normal pregnancy models: in the vinous samples of normal pregnancy models, the expression of p53 was positively correlated with the expression of MDM2, VEGF and HIF-la $(r=0.31 ; r=0.48 ; r=0.67)$, and the relationship was significant $(\mathrm{P}=0.03 ; \mathrm{P}$ $=0.003 ; \mathrm{P}<0.001)$. The expression of MDM2 was positively correlated with the expression of VEGF $(r=0.23)$, but the relationship was not significant $(\mathrm{P}=0.11)$; and negatively correlated with the expression of HIF-la $(\mathrm{r}=-0.03)$, but the relationship was not significant $(\mathrm{P}=0.84)$. The expression of HIF-la was positively correlated with the expression of VEGF $(r=0.35)$, and the relationship was significant $(P=0.01)$. Conclusion: angiogenesis reduces in villous samples of spontaneous abortion mouse model, P53 and MDM2 involve in angiogenesis in villous samples, unlikely p53, and MDM2 have effects on normal early pregnancy villous angiogenesis and when the cell DNA damages or hypoxia exacerbates, it can induce high expression of p53, MDM2, inhibit angiogenesis in villous samples in early pregnancy. P53, MDM2 generegulate villous trophoblast cell growth by adjusting expression of HIF-1a and VEGF gene, finally influences pregnancy.
\end{abstract}

Keywords: p53; MDM2; VEGF; HIF-la; Spontaneous Abortion

\section{Introduction}

Spontaneous abortion is one of the common obstetric pathological pregnancies, its etiology is complex. In addition to anatomy, endocrine, infection, chromosome, genetic factors, of which $40 \%-80 \%$ unexplained spontaneous abortion may be associated with immune factors. Although studies have confirmed the occurrence of spontaneous abortion may be associated with excessive cell

\footnotetext{
${ }^{*}$ Corresponding author.
}

apoptosis, at present its specific regulation mechanism lack systematic and thorough research. The related genes of p53, MDM2 play an important role in regulating apoptosis, occurrence mechanism of spontaneous abortion. The spontaneous abortion mouse models were chosen for the study. We applied q Real-time PCR method to detect genes expression levels in villous samples of spontaneous abortion mouse models and normal pregnancy models, discussed the effect of p53, MDM2 on pregnancy, studied the mechanism of spontaneous abortion. 


\section{Material and Method}

1) Experiment animals and reagent

Female CBA/J, male DBA/2, BALB/C three mouse, clean 8 - 10 weeks of age, were bought in Shanghai Science Development Company.p53, MDM 2 Specific primer, p53, MDM2 VEGF and HIF-1a antibody, DNA color-developing agent all related reagents were purchased at Nanjing University.

2) Model preparation and grouping

Establish a mouse model, the abortion-prone CBAXD$\mathrm{BA} / 2$ were established as the model of spontaneous abortion and the non-abortion-prone CBAXBALB/c prone as the model of normal pregnancy, and detected Vaginal suppository as pregnancy $0 \mathrm{~d}$.

\section{3) Specimen handling}

At the 13th day, two groups of pregnant mouse models were put to death. We collected a partial villous samples (samples came from the normal survival embryonic) and stored them at $-70^{\circ} \mathrm{C}$. Another small part fixed with $4 \%$ formalin in room temperature was immobilized for 24 hours. Serial sections, the thickness about $4 \mu \mathrm{m}$, put in the 60 degrees oven, dried after $72 \mathrm{~h}$ for backup.

4) Applied q Real-time PCR method to detect the mRNA expression levels of p53, MDM2, VEGF and HIF-la in villous samples of spontaneous abortion mouse models and normal pregnancy models

Did strictly as agent instructions said. Reaction procedure: pre degenerated at $95^{\circ} \mathrm{C}$ for $10 \mathrm{~min}$, degenerated at $95^{\circ} \mathrm{C}$ for 15 sec. Annealed at $58^{\circ} \mathrm{C}$ for $20 \mathrm{sec}$ (p53, MDM2, HIF-1a), Annealed at $61^{\circ} \mathrm{C}$ for 20 sec (VEGF), extended at $72^{\circ} \mathrm{C}$ for $10 \mathrm{sec}$, circulated a total of 45 times, at each end of circulation amplification detected fluorescence signal. Subsequently doing melting curve analysis, a slow heating procedure: from $6^{\circ} \mathrm{C}$ up to $95^{\circ} \mathrm{C}$ at rate of $0.1^{\circ} \mathrm{C} / \mathrm{sec}$, then cooled down to $40^{\circ} \mathrm{C}$, at the same time, continuously monitored fluorescence signal changing, in the progress of double chain degenerating gradually, drew melting curve of products, in order to understand the specificity of sample amplification, made sure measurement results were precise and reliable. After the reaction was finished, the computer according to amplification of the quantitative standard template automatically drew standard curve. According to the respective standard curve, the software automatically calculated accurate content of the sample to be tested in the gene or housekeeping gene.

\section{Statistical Methods}

Used SPSS12.0 statistical software to analyze data, Spearman rank relation coefficient to analyze the relationship of the mRNA expression level of p53, MDM2, VEGF and HIF-la in vinous samples. \pm SD was used as measurement data, applied variance homogeneity test and single factor variance analysis to compare measurement data group mean. The two groups of measurement data were compared by student T-test. The two each other in many groups were compared with SNK-q test. Counted data using $\times 2$ test. $\mathrm{P}<0.05$ was considered obviously different.

\section{Results}

The relationship of the mRNA expression level of $\mathrm{p} 53$, MDM2, VEGF and HIF-la in vinous samples of spontaneous abortion mouse models were shown in Table 1. The study found the relationship of the mRNA expression level of p53, MDM2, VEGF and HIF-la in vinous samples of spontaneous abortion mouse models: in the villous samples of spontaneous abortion mouse models, the expression of p53 was positively correlated with the expression of MDM2, HIF-la ( $r=0.35 ; r=0.63)$, and the relationship was significant $(\mathrm{P}=0.01 ; \mathrm{P}<0.001)$; but negatively correlated to the expression of VEGF ( $\mathrm{r}=$ $-0.30)$, and the relationship was significant $(P=0.03)$. The expression of MDM2 was positively correlated with the expression of HIF-la $(r=0.28)$, and the relationship was significant $(\mathrm{P}=0.04)$; and negatively correlated with the expression of VEGF ( $\mathrm{r}=-0.08)$, but the relationship was not significant $(\mathrm{P}=0.57)$. The expression of HIF-la was negatively correlated with the expression of VEGF $(\mathrm{r}=-0.37)$, and the relationship was significant $(\mathrm{P}=$ 0.007). Therefore, in the vinous samples of spontaneous abortion mouse models the increased expression of p53, MDM2 and HIF-la could make expression of VEGF decrease.

The relationship of the mRNA expression level of p53, MDM2, VEGF and HIF-1 a in vinous samples of normal pregnancy models were shown in Table 2: in the vinous samples of normal pregnancy models, the expression of p53 was positively correlated with the expression of MDM2, VEGF and HIF-la $(r=0.31 ; r=0.48 ; r=0.67)$, and the relationship was significant $(\mathrm{P}=0.03$; $\mathrm{P}=0.003$; $\mathrm{P}<0.001)$.

The expression of MDM2 was positively correlated with the expression of VEGF ( $r=0.23)$, but the relationship was not significant $(\mathrm{P}=0.11)$; and negatively correlated with the expression of HIF-la $(r=-0.03)$, but the relationship was not significant $(P=0.84)$. The ex-

Table 1. The relationship of the mRNA expression level of p53, MDM2, VEGF AND HIF-la in vinous samples of spontaneous abortion mouse models.

\begin{tabular}{cccccccccc}
\hline \multirow{2}{*}{ TABLE HEAD } & \multicolumn{2}{c}{ P53 } & \multicolumn{2}{c}{ MDM2 } & \multicolumn{2}{c}{ VEGF } & \multicolumn{2}{c}{ HIF-1A } \\
\cline { 2 - 9 } & $\boldsymbol{r}$ & $\mathrm{P}$ & $\boldsymbol{r}$ & $\mathrm{P}$ & $\boldsymbol{r}$ & $\mathrm{P}$ & $\boldsymbol{r}$ & $\mathrm{P}$ \\
\hline P53 & 1.00 & - & 0.35 & 0.01 & -0.30 & 0.03 & 0.63 & $<0.001$ \\
MDM2 & - & - & 1.00 & - & -0.08 & 0.57 & 0.28 & 0.04 \\
VEGF & - & - & - & - & 1.00 & - & -0.37 & 0.07 \\
\hline
\end{tabular}


Table 2. The relationship of the mRNA expression level of p53, MDM2, VEGF and HIF-1a in vinous samples of normal pregnancy models.

\begin{tabular}{ccccccccc}
\hline & \multicolumn{2}{c}{ P53 } & \multicolumn{2}{c}{ MDM2 } & \multicolumn{2}{c}{ VEGF } & \multicolumn{2}{c}{ HIF-1A } \\
\cline { 2 - 10 } & $\boldsymbol{r}$ & $\mathrm{P}$ & $\boldsymbol{r}$ & $\mathrm{P}$ & $\boldsymbol{r}$ & $\mathrm{P}$ & $\boldsymbol{r}$ & $\mathrm{P}$ \\
\hline P53 & 1.00 & - & 0.31 & 0.03 & 0.48 & 0.003 & 0.67 & $<0.001$ \\
MDM2 & - & - & 1.00 & - & 0.23 & 0.11 & -0.03 & 0.84 \\
VEGF & - & - & - & - & 1.00 & - & 0.35 & 0.01 \\
\hline
\end{tabular}

pression of HIF-la was positively correlated with the expression of VEGF ( $r=0.35)$, and the relationship was significant $(P=0.01)$. Therefore, in vinous samples of normal pregnancy models, the increased expression of p53, MDM2 and HIF-la could make expression of VEGF increase, too.

\section{Discussion}

Apoptosis is a process of regulating body growth, maintaining homeostasis, actively removing damaged and nonrequired cells controlled by genes. The imbalanced expression of related genes about cell apoptosis probably leading to various pathological pregnancy is one of the important reasons, placenta is the important position directly connecting the maternal and infant in vivo, it's normal development is crucial to maintain normal pregnancy, in order to better adapt to the needs of the fetus, placenta reconstructs tissue by villous apoptosis and proliferation. There exists trophoblastic cells apoptosis to some degree in normal pregnancy, in the process of embryo development, moderate apoptosis facilitates villous blood lumen formation and the development of villus in early pregnancy [1-3]. The progress of embryo development and placenta germination is in a relatively hypoxic environment. In the early pregnancy (first 12 weeks of pregnancy), trophoblastic cells adapting to hypoxia environment is the key to successful pregnancy.

In the progress of pregnancy, with the level of oxygen changing, angiogenesis of placenta trophoblastic vascular network will change, too [4]. Trophoblastic cells come from embryonic trophectoderm, they play an important role in process of embryo implantation, its proliferation, functional disorders are closely correlated with occurrence and development of multiple pregnancy diseases. Successful pregnancy depends on right fetal placenta vascular development in trophic layer, only in this way can the oxygen and nutrients translate from the gap of trophoblastic cells to the fetus [5]. In early embryo development, angiogenesis is the most basic condition of embryo implantation and development of uterine endometriumt, angiogenesis requires multiple factors interaction, including a variety of hormones, growth factors, soluble extracellular matrix molecules [6]. VEGF is one of the most important angiogenesis regulation factors [7], the normal expression of VEGF is an important factor to maintain pregnancy. The decreased expression of VEGF may be a danger signal about abortion, its implantation is a complex, ambiguous process, VEGF has a crucial role in the process of blastocyst implantation and placenta growth and development [8] VEGF is a highly conserved glycoprotein, composed of two identical subunits (Mr23,000) cross-linking together with disulfide, the molecular weight is between 34,000 - 45,000, Isoelectric point 8.5. VEGF has four different variants, the gene is located on the sixth chromosome long arm, including the alternative splicing 8 exons and 7 introns, the biological activity of the four VEGF is similar, in human cells, the amino acid residues are 121, 165, 189 and 206. The variants about 121, 165 of amino acid residues are easy to arrive the target cell, pregnancy is inseparable from the rich blood supply from implantation to placenta formation, then to delivery, and is inseparable from the angiogenesis, permeability changing. When the fertilized egg implantation, blastocyst is not closed with maternal blood, human blastocyst development is in a relatively hypoxic state, so hypoxia can make VEGF activity increased [9], VEGF can promote angiogenesis, thereby improving blood flow, increasing supply of oxygen in hypoxia tissue [10]. Shiraish [11] uses immunological technique to discover VEGF expression of syncytiotrophoblast and extravillous trophoblast cells during the pregnancy process, and VEGF also expresses in villous stromal and decidual cells. The expression of VEGF is not balanced in early pregnancy in syncytiotrophoblast cells, at near vascular buds the concentration is higher, morphological analysis shows masculine expression area of VEGF is villous blood vessel formation region, explaining placenta trophoblasts generating VEGF to promote the development of placenta vascular network. Use immunohistochemical method to find the VEGF expression decreases in trophoblastic cell in patients with missed abortion. Vuorela [12] uses immunohistochemical method to find the VEGF expression decreases in trophoblastic cells in patients with missed abortion.

Fertilized ovum from implantation to delivery, oxygen concentration of placenta is changing. Human placenta development is divided into two stages, early stages of pregnancy (first 3 months of pregnancy), is placenta formation stage, the oxygen concentration of placenta site is low. When embryo inserts endometria, oxygen concentration is only 10 - $15 \mathrm{mmHg}$, embryo relies on the secretion of uterine gland to provide nutrients, increased flow of uterine placenta blood is closely related to the changes of trophoblast penetrating endometrium and uterine spiral artery. First 12 weeks of pregnancy, the villous space bending and uterine spiral artery diameter being relatively narrow, limit blood flowing into the villous space, the site of placenta implantation is a low oxygen concentra- 
tion. After 12 weeks of pregnancy, trophoblastic cell immerse into uterine spiral artery, remodel the vascular layer, make narrow artery expanded, placenta blood flow increased, corresponding placenta site oxygen concentrations also increased [13]. Therefore, early trophoblast development is in hypoxia environment, hypoxia has effect on trophoblast immersion.

At present people have reached an agreement, placenta trophoblast angiogenesis is strictly regulated by the oxygen concentration. Hypoxia may lead to hypoxic cell stressed, performing for the role of hypoxia in cells has effect on oxygen sensors, initiates intracellular signal transduction pathways, activate as a transcription HIF-1 a, increased HIF-I a expression [14].

HIF-1 is a major hypoxia-inducible transcription factor, with one a subunit and one B subunit, HIF-1a is an adjustable function unit, regulated by the oxygen concentration, in recent years, studies have shown that HIF-1a under certain condition has effects on apoptosis. Under hypoxia state, HIF-1 makes higher expression of P53 and inducts p21 synthesis. Suppress activity of cell cycle dependent kinase, makes cells stay in the G1 period, promotes cell apoptosis, HIF-1 as the organism regulation factor under hypoxic conditions, mediates hypoxia-induced apoptosis, hypoxia can increase the wild-type embryonic stem cell (ES, HIF-1a) apoptosis, but can not promote embryonic stem cells (ES, HIF-1 $\beta$ ) of gene deletion apoptosis, they incubated tumor derived from embryonic stem cell, also showed that HIF-1a genotype tumor apoptosis less than HIF- $1 \beta$ genotype hypoxic inducing apoptosis is mediated by HIF-1. Moritz also found early cell apoptosis is related to high expression of HIF1 when pancreas transplantation is for type I diabetes.

VEGF is the major transcription factor of HIF-1a. Under anoxic state, the high expression of HIF-la can induce higher expression of VEGF, but the too high expression of HIF-la could inhibit angiogenesis [14]. HIFla induces apoptosis in hypoxia lying in p53 protein through combining the oxygen degradation domain, ODD and HIF-1 a, compound formation, enhance the stability of p53. The p53 protein is a short-lived protein, the intracellular concentration is strictly regulated by the negative accommodation factor mouse double minute gene 2 (MDM2) gene [15-17]. MDM2 is an important intracellular regulating p53 concentration and its activity, as p53 important activity molecules to involved in cell growth inhibition, apoptosis, regulation of cell cycle process. The expression of wild type P53 protein can stimulate high expression of MDM2 protein. Based on the p53 and MDM2 negative feedback loop, study found increased MDM2 protein can inhibit p53 protein function, thus increasing the negative feedback loop function. Factors that can affect the p53, MDM2 or P53-MDM2 interaction have an important effect on for cell fate (apoptosis, cell cycle arrest, etc.) [18]. The changing of MDM2 gene promoter sequence change can alter the expression of MDM2 protein. The expression of MDM2 protein improves, make p53 reduce stability, therefore change the apoptotic reaction of p53 mediating on DNA damage. $[17,19]$.

If MDM2 identifies target p53, meanwhile combines with HIF-1a subunit, it can mediate HIF-1 a degradation, thereby affecting the expression of VEGF. Our study found, the expression of p53, MDM2 and HIF-1 are higher in villous samples of spontaneous abortion mouse models than normal pregnancy models, however the expression of VEGF is lower in villous samples of spontaneous abortion mouse models than normal pregnancy models. We hypothesized that in the progress of normal early villous samples development, Chorionic villus in is the hypoxic microenvironment, hypoxia makes the expression of HIF-la gene higher, leading to the lower expression of VEGF, thus prompting villus angiogenesis, ensuring the success of early pregnancy. However in the progress of spontaneous abortion, Chorionic villus cells suffer a more severe hypoxia and DNA damage, make higher expression of p53, make higher expression of MDM2 by the positive feedback regulation, and hypoxia can cause expression of HIF-la increased further, but MDM2 distinguishes target p53, meanwhile combines HIF-1 a subunit, instead mediates HIF-1 a degradation, making the expression of VEGF lower and angiogenesis decreased, at the same time making cell cycle arrested, apoptosis increased, eventually leading to the occurrence of spontaneous abortion. Therefore, based on the study of chorionic, we hypothesized that the p53, MDM2 gene plays a role in regulating during early pregnancy villous samples development, finally influencing the pregnancy.

\section{REFERENCES}

[1] M. Jerzak and P. Bischof, "Apoptosis in the First Trimester Human Placenta: The Role in Maintaining Privilege at the Matemal-Foetal Interface and the Trophoblast Remodeling," The European Journal of Obstetrics \& Gynecology and Reproductive Biology, Vol. 100, 2002, pp. 138142. http://dx.doi.org/10.1016/S0301-2115(01)00431-6

[2] D. Michael and M. Oren, "The p53-Mdm2 Module and the Ubiquitin System,” Cancer Biology, Vol. 13, 2003, pp. 49-58. http://dx.doi.org/10.1016/S1044-579X(02)00099-8

[3] G. Beckman, R. Birgander, A. Sjalander, et al., "Is p53 Polymorphism Maintained by Natural Selection?” Human Heredity, Vol. 44, 1994, pp. 266-270. http://dx.doi.org/10.1159/000154228

[4] L. V. J. Schaffer, C. Breymann, M. Gassmann and H. H. Marti, "Preserved Placental Oxygenation and Development during Severe Systemic Hypoxia," The American Journal of Physiology Regulatory, Integrative and Comparative Physiology, Vol. 290, No. 3, 2006, pp. 844-851. http://dx.doi.org/10.1152/ajpregu.00237.2005 
[5] A. C. K. Ajakumar, "Expression, Ontogeny, and Regulation of Hypoxia-Inducible Transcription Factors in the Human Placenta," Biology of Reproduction, Vol. 63, No. 2, 2000, pp. 559-569. http://dx.doi.org/10.1095/biolreprod63.2.559

[6] D. E. F. J. Ingber, "Mechanochemical Switching between Growth and Differentiation during Fibroblast Growth Factor-Stimulated Angiogenesis in Vitro: Role of Extracellular Matrix,” The Journal of Cell Biology, Vol. 109, No. 1, 1989, pp. 317-330.

[7] M. D. L. D. Mueller, E. Garrett and R. N. Taylor, "Neutrophils Infiltrating the Endometrium Express Vascular Endothelial Growth Factor: Potential Role in Endometrial Angiogenesis," Fertility and Sterility, Vol. 74, No. 1, 2000, pp. 107-112.

[8] S. SK, “Angiogenesis and Implantation,” Human Reproduction, Vol. 15, No. S6, 2000, pp. 59-66.

[9] L. O. A. Hefler, P. Husslein, C. Kainz and C. Tempfer, "Vascular Endothelial Growth Factor Serum Levels in Pregnancy and Preeclampsia,” Acta Obstetricia et Gynecologica Scandinavica, 2000, pp. 77-78.

[10] H. H. R. W. Marti, “Angiogenesis in Ischemic Disease,” Vol. 82, 1999, pp. 44-52.

[11] S. N. K. aishi, N. Kinukawa, H. Nakano and K. Sueishi, "Immunohistochemical Localization of Vascular Endothelial Growth Factor in the Human Placenta,” Placenta, Vol. 17, No. 2-3, 1996, pp. 111-121.

[12] P. C. O. Vuorela, M. Tulppala and E. Halmesmaki, "VEGF, Its Receptors and the Tie Receptors in Recurrent Miscarriage," Molecular Human Reproduction, Vol. 6, No. 3, 2000, pp. 276-282.
[13] G. J. H. J. Burton and A. L. Wetson, "Maternal Arterial Connections to the Placectal Intervil Lous Space during the First Trimester of Human Pregnancy,” American Journal of Obstetrics \& Gynecology, Vol. 181, 1999, p. 71.

[14] H. D. Z. J. Skinner, J. Fang, F. Agani and B. H. Jiang, "Vascular Endothelial Growth Factor Transcriptional Activation Is Mediated by Hypoxia-Inducible Factor Lalpha, HDM2, and p74S6K1 in Response to Phosphatidylinositol 3-Kinase/AKT Signaling," The Journal of Biological Chemistry, Vol. 279, No. 44, 2004, pp. 45643-51.

[15] M. Thomas, A. Kalita, S. Labrecque, et al., "Two Polymorphic Variants of Wild-Type p53 Differ Biochemically and Biologically,” Molecular and Cellular Biology, 1999, pp. 1092-1100.

[16] P. Dumont, J. I. Leu, A. Cr. Della Pietra, et al., “The Codon 72 Polymorphic Variants of p53 Have Markedly Different Apoptotic Potential,” Nature Genetics, Vol. 33, 2003, pp. 357-365.

[17] G. L. Bond, W. Hu, E. E. Bond, et al., “A Single Nucleotide Polymorphism in the MDM2 Promoter Attenustes the p53 Tumor Suppressor Pathway and Accelerates Tumor Formation in Humans,” Cell, Vol. 119, 2004, pp. 591602.

[18] D. Michael and M. Oren, "The p53-Mdm2 Module and the Ubiquitin System,” Cancer Biology, Vol. 13, 2003, pp. 49-58.

[19] Y. Hong, X. Zhang, et al., "The Role of p53 and MDM2 Polymorphisms in the Risk of Esophagel Squamaous Cell Carcinoma,” Cancer Research, Vol. 65, 2005, pp. 95829587. 